\title{
Obesidad y Pobreza: marco conceptual para su análisis en latinoamérica
}

\section{Obesity and Poverty: conceptual references for its analysis in Latin America}

\author{
Dixis Figueroa Pedraza \\ Doutor em Nutrição. Pesquisador do Departamento de Enfermagem \\ e Núcleo de Estudos e Pesquisas Epidemiológicas (NEPE). Univer- \\ sidade Estadual da Paraíba. \\ Endereço: Rua Ricardo Wagner, 138, apto. 105, Bodocongó, CEP \\ 58429-110, Campina Grande, PB, Brasil. \\ E-mail: dixisfigueroaळgmail.com
}

\section{Resumen}

La obesidad tiene una etiología multifactorial en la que los factores de mayor fuerza se relacionan con los estilos de vida. El incremento tan abrupto de la obesidad y su gran extensión obedecen principalmente a cambios importantes en la alimentación de la población, al patrón de actividad física y a otros factores de índole sociocultural, todo lo cual se ha manifestado en un proceso de transición nutricional. Las tendencias de aumento y de las diferencias sociales en la obesidad, hacen que las minorías y aquellos estratos de la población con menor nivel educativo y socioeconómico puedan ser los más afectados. En los pobres la obesidad se asocia, entre otros, a episodios de desnutrición en la edad temprana, incluso durante la vida intrauterina, a factores alimentarios, socioculturales y de género. La relación entre la obesidad y la condición socioeconómica es muy variable y compleja, ya que la asociación es diferente en contextos socioeconómicos distintos y puede quedar disimulada por varios factores (ejercicio físico, dieta, factores psicológicos, herencia, factores socioculturales) que influyen en la asociación. Tanto el nivel socioeconómico podría condicionar la presencia de obesidad, como la obesidad podría tener consecuencias sociales en el individuo (si la movilidad social es el factor más relevante, es la obesidad la que condicionaría la posición social del individuo). Los argumentos tratados son suficientes para justificar la importancia del problema en Latinoamérica. De esta manera, debe representar un desafío, principalmente para investigadores y aquellos que toman decisiones, traducible en priorizar la obesidad en condiciones de pobreza.

Palabras-clave: Obesidad; Pobreza. 


\section{Abstract}

Obesity has a multifactorial etiology in which the most important factors are related to lifestyle. High prevalence of obesity is basically caused by important changes in nourishment, in the pattern of physical activity and by other sociocultural factors, which have produced a nutritional transition process. Due to the trends of increase and of social differences in obesity, the minorities and populations with lower educational and socioeconomic level can be the most affected groups. In poor people, obesity is associated, among others, with malnutrition episodes in childhood, even during intrauterine life, and also with alimentary, sociocultural and gender factors. The relationship between obesity and socioeconomic condition is very changeable and complex, since the association is different in distinct socioeconomic contexts and it may remain hidden by several factors (physical exercise, diet, psychological factors, heredity, sociocultural factors) that influence the association. The socioeconomic level might determine the presence of obesity, and obesity, in turn, might cause social consequences to the individual (if social mobility is the most relevant factor, obesity is the one that would determine the individual's social position). The arguments proposed here are sufficient to justify the importance of the problem in Latin America. Thus, it must represent a challenge, principally for researchers and decision makers, translatable in prioritizing obesity in poverty conditions.

Keywords: Obesity; Poverty.

\section{Introducción}

El estado nutricional es definido como la disponibilidad y utilización de nutrientes y energía a nivel celular. Así, la situación nutricional es normal cuando la oferta de nutrientes a través de la alimentación se corresponde con las necesidades metabólicas normales y sus variaciones (anabolismo/catabolismo) inducidas por sobrecargas fisiológicas, ocupacionales y patológicas. Esto conduce a dos alternativas posibles:

1. Si la disponibilidad de energía y nutrientes es inferior a las necesidades, tendremos como resultado el desarrollo de enfermedades carenciales / malnutrición por defecto;

2. Si la oferta excede las exigencias biológicas, con valores superiores a los niveles tolerables, tendremos como resultado el desarrollo de patologías por excesos nutricionales / malnutrición por exceso, con destaque para la obesidad por ser la principal epidemia de los tiempos modernos (Batista Filho, 2003).

Se puede definir la obesidad como un exceso de grasa corporal (tejido adiposo), acompañado por un aumento en la relación entre el peso y la estatura, en la frecuencia de algunas enfermedades y mortalidad de las personas que la padecen. Operativamente, puede considerarse la obesidad como la condición en la cual el Índice de Masa Corporal es mayor que 3o. La obesidad se asocia a un exceso en el número de adipositos, a un desproporcionado tamaño de los mismos, o a ambos procesos. El número final de adipositos queda establecido al término de la adolescencia (Hodalgo y col., 2002).

La obesidad tiene una etiología multifactorial. Es el resultado de la conjunción de factores biológicos, genéticos y ambientales. En general, los factores determinantes más estudiados de la obesidad están relacionados con el estilo de vida, especialmente el binomio alimentación y actividad física (Ferreira y Wanderley, 2009). La obesidad no debe ser considerada únicamente como un disturbio producido por el desequilibrio entre las necesidades energéticas y la alimentación, si no como un disturbio nutricional que puede coexistir con deficiencias de micronutrientes y otras enfermedades carenciales, particularmente en los grupos socioeconómicos más vulnerables. La asociación de la obesidad con el nivel socioeconómico, y otros factores como el género, edad y etnicidad es compleja y dinámica (Peña 
y Bacallao, 2006). El presente artículo se propone analizar la complejidad de la asociación entre obesidad y nivel socioeconómico, desde una perspectiva de salud pública, de forma tal que su comprensión en la región se refrita en investigaciones que ayuden a profundizar los efectos al compararse realidades diferentes.

\section{Magnitud del Problema y Tendencias}

Estudios en el mundo entero muestran una tendencia creciente en las prevalencias de obesidad y sobrepeso. El aumento progresivo y acelerado es observado tanto en países o regiones desarrollados como en desarrollo, en ambos sexos y en todos los grupos de edad. Referido a la edad, los estudios reflejan que, en general, existe un pico en la edad adulta, y una incidencia menor en la niñez y la adolescencia (Peña, 20o1; Eberwine, 2002, p. 1).

La obesidad ha alcanzado proporciones de una epidemia global. Las tasas de obesidad, como mínimo, se han triplicado desde 1980 en algunas partes de América del Norte, Europa del Este, Oriente Medio, las Islas del Pacífico, Australia y China. La región latinoamericana no está ajena a esta tendencia y el aumento en las cifras de obesidad se ha hecho más evidente a medida que los países mejoran su ingreso económico'. La evolución de la obesidad ha sido observada en países como Chile, México, Brasil, Argentina, Perú, Colombia, Guatemala y Bolivia (Peña y Bacallao, 2001, 2005).

En Brasil, por ejemplo, estudios nacionales realizados en los años 1974-1975 y 1989 indicaron un aumento en las prevalencias de obesidad de $2 \%$ a $5 \%$ y de $7 \%$ a $12 \%$, en hombres y mujeres, respectivamente. Si consideramos el último de los anteriores estudios, 6,8 millones ( $8 \%$ ) de individuos adultos presentaron obesidad y cerca de 27 millones (32\%) presentaron algún grado de exceso de peso. De estos, aproximadamente 11 millones eran hombres y 16 millones mujeres, correspondiendo a porcentajes en torno de $27 \%$ y $38 \%$ de la población masculina y femenina, respectivamente. La prevalencia del agravo más acentuado en el grupo femenino, fue especialmente más fuerte en las mujeres de baja renta (Ferreira y Magalhães, 2006).

Estudios nacionales realizados en los años 1988 y 1999 en México demostraron que la prevalencia de obesidad en su población adulta femenina aumentó de $9,4 \%$ a $24,4 \%$ (Rivera y col., 2002, p. 123). Tendencias similares a la anterior se han observado en Costa Rica, Barbados y algunas otras ciudades del Caribe. Una característica común encontrada son las altas tasas de obesidad en mujeres de bajos ingresos y el aumento de la obesidad en niños menores de cinco años de las ciudades más pobres (De Onis y Blössner, 2000, p. 1032).

Los estudios realizados en Chile también apuntan un aumento alarmante en las cifras de obesidad. Los datos nacionales, utilizando como indicador el Índice de Masa Corporal de acuerdo a los patrones CDC, muestran un aumento en las prevalencias que pasaron de $6 \%$ en 1987 a 18\% en 2000 (Uauy y col., 2001, p. 893S).

Considerando el nivel socioeconómico, varias investigaciones han mostrado una tendencia ascendente de las diferencias sociales en la obesidad, principalmente en mujeres. Este fenómeno, de extraordinaria importancia para la epidemiología y la salud pública, nos sitúa ante un problema de desigualdad social complejo, en el que se entremezclan factores sociales y, probablemente, biológicos, y en el que la desventaja de pertenecer a un grupo de bajo nivel socioeconómico y de ser mujer tiene consecuencias importantísimas para la salud (Gutiérrez Fisac y col., 1996, p. 351; Peña y Bacallao, 2005).

El aumento acelerado de las cifras de obesidad en la región, ha resultado en un consenso internacional que coloca la enfermedad como un importante problema de salud pública cuyo estudio se justifica por las siguientes razones (Peña y Bacallao, 2005)2:

- La ganancia de peso corporal está asociada a un aumento de la morbilidad y mortalidad. La obesidad es un factor de riesgo de varias enfermedades crónicas no transmisibles asociadas a la nutrición (ECNT), algunas de las cuales son causa importante de muerte en adultos, por ejemplo, la enfermedad isquémica del corazón,

1 ISUNZA VERA, A. La obesidad en la pobreza: violación al derecho a la alimentación. 2004. Monografía (Postgrado a distancia en obesidad) - Universidad de Favaloro, Buenos Aires, 2004.

2 VÁZQUEZ SÁNCHEZ, R.; LÓPEZ ALEMANY, J. Obesidad: la epidemia del siglo XXI. Revista Española de Economía de la Salud, Madrid, v. 1, n. 3, p. 34-36, 2002. Disponível em: <http://www.economiadelasalud.com/Ediciones/o3/PDF/o3Analisis_Introduccion.pdf>. Acesso em: 1 dez. 2006. 
la diabetes mellitus no insulinodependiente o de tipo II (DMNID), la hipertensión arterial, algunos tipos de cáncer, la osteoartritis y la osteoporosis, además de otras enfermedades crónicas como colecisto litiasis, enfermedades osteomusculares, respiratorias y psicológicas.

- Resulta muy difícil mantener la pérdida de peso en personas con sobrepeso.

- Las medidas para prevenir la obesidad, especialmente las que tienden a mejorar la calidad de la alimentación y incrementar la actividad física sistemática, coinciden en gran parte con las medidas para prevenir otras ECNT.

- La obesidad es un indicador útil para la vigilancia, pues es fácil detectarla mediante las mediciones antropométricas tradicionales.

\section{Factores que Justifican la Tendencia de Aumento de la Obesidad}

El incremento tan abrupto de la obesidad que ha ocurrido en las últimas décadas, así como su gran extensión, obedece principalmente a cambios importantes en la alimentación de la población, al patrón de actividad física y a otros factores de índole sociocultural (Peña y Bacallao, 2001, p. 75). Estos aspectos pueden ser abordados de la siguiente manera:

I - Cambios más relevantes en los patrones alimentarios (Peña y Bacallao, 20o1, p. 75; Figueroa Pedraza, 2003; Popkin, 1998, p. 5)

- Alto consumo de alimentos de alta densidad energética y bajo contenido de nutrientes.

- Alto consumo de azúcares y carbohidratos refinados.

- Alto consumo de grasas, principalmente saturadas, ácidos grasos trans y colesterol.

- Alto consumo de bebidas alcohólicas.

- Pobre consumo de vegetales y frutas frescas.

- Pobre consumo de carbohidratos complejos y fibra.

- Aumento del tamaño de las raciones de alimentos, especialmente en restaurantes y "cadenas de alimentos rápidos”.

- Aumento del consumo de alimentos procesados que conllevan a un aumento del consumo de carbohidratos simples (principalmente azúcar), aumento del consumo de grasas (principalmente saturada) y disminución en el consumo de carbohidratos complejos y fibra.
II - Reducción de la actividad física (Peña y Bacallao, 2001, p. 75; Popkin, 200o; Popkin, 2001, p. 871S)

- Reducción del trabajo físico debido a adelantos tecnológicos.

- Uso cada vez mayor de transporte automotor.

- Automatización de los vehículos y reducción del gasto energético en la operación de maquinarias y vehículos (cierre y ventanillas electrónicas, por ejemplo).

- Uso de ascensores y "escaleras rodantes".

- Reducción del tiempo dedicado a jugar al aire libre, por inseguridad, por ejemplo, y preferencia por juegos electrónicos y la televisión.

\section{III - Aspectos socioculturales (Peña y Bacallao, 2001,} p. 75)

- Comprensión de la obesidad como expresión de salud y no de enfermedad.

- Comprensión de la obesidad como sinónimo de afluencia en algunas culturas.

- Aceptación de la obesidad como expresión del patrón cultural de belleza influenciado por los medios de comunicación.

\section{IV-Procesos de transición asociados a los anteriores factores (Popkin, 2001, p. 871S) ${ }^{1}$}

- Transición demográfica.

- Transición epidemiológica.

- Transición nutricional.

La transición demográfica es un proceso por el que atraviesan o han atravesado casi todos los países del mundo. Se refiere al transito de un régimen caracterizado por niveles de mortalidad y fecundidad elevados, y sin control, a otro de niveles bajos y controlados, acompañados por un aumento de la esperanza de vida (Popkin, 1998, p. 5)'.

La transición epidemiológica enfoca el cambio en los patrones de salud y enfermedad. Se refiere al transito de poblaciones con altas prevalencias de enfermedades infecciosas, por pobres condiciones ambientales, a poblaciones con predominio de enfermedades crónicas. El proceso de transición epidemiológica está condicionado por la transición demográfica, por cambios socio-económicos y por el proceso de urbanización-modernización (Rivera y col., 2002, p. 123; Popkin, 200o; Albala y Vio, 2006). 
La transición nutricional describe los cambios en el régimen alimentario, en el patrón de trabajo, en la actividad física y en el tipo de recreación. Se refiere al transito de poblaciones con altas prevalencias de desnutrición a poblaciones con predominio de enfermedades crónicas no transmisibles relacionadas con la nutrición. El proceso de transición nutricional está condicionado por las transiciones demográfica y epidemiológica, por los cambios socio-económicos, como el aumento de los ingresos, por el proceso de urbanización-modernización, por cambios en las características de ocio y trabajo (avance tecnológico) y por la influencia de los medios de comunicación de masas y el marketing de alimentos ${ }^{3}$ (Popkin, 1998, p. 5; Popkin, 200o; Popkin, 2001, p. 871S).

\section{Breve Caracterización de la obesidad en la Pobreza}

La pobreza es aquella situación en la cual la población padece escasez de recursos y un acceso limitado y dependiente a los mismos, existe cuando los individuos o grupos no están en condiciones de satisfacer adecuadamente sus necesidades básicas: alimentos, vida social y cultural, educación primaria, salud, condiciones y ambiente favorable para la vida (vestimenta, habitación, agua, aire, etc.). En términos económicos, se puede considerar como medida de la pobreza la capacidad de comprar dos canastas básicas de alimentos con la renta devengada. Así, la pobreza normalmente se acompaña de hambre, malnutrición, desigualdad y injusticia social ${ }^{1}$.

A pesar de que la pobreza normalmente está relacionada con aspectos económicos, su abordaje es mucho más complejo. Es conocido que para la erradicación de la pobreza no basta con el crecimiento económico de un país. Existe un punto en el cual los aumentos en el crecimiento no logran disminuir las condiciones de pobreza de la población. Desde este punto de vista se puede distinguir entre pobreza coyuntural y pobreza estructural (López, 2007).

La pobreza coyuntural es aquella que depende de las condiciones de producción y empleo de la economía; por lo tanto, en periodos de auge económico la demanda de trabajo aumenta, y en consecuencia, tanto el nivel de salarios, como el empleo crecen, generando beneficios sociales en términos de los ingresos familiares y la disminución de la pobreza. La pobreza estructural es aquella que no tiene una correlación importante con el crecimiento económico. En general, la explicación tiene que ver con la particularidad que tienen las características del mercado de trabajo, las características psicosociales, y de capital social, entre otros aspectos, de los "pobres estructurales" que quedan atrapados en círculos viciosos de pobreza con condiciones que se retroalimentan y empeoran su situación en el tiempo. Entre estas condiciones se encuentra el trabajo infantil, analfabetismo, desnutrición, criminalidad, calificación y desplazamiento forzado. De esta manera, queda claro la complejidad del problema, haciendo con que ninguna forma de crecimiento logra absorber la pobreza estructural, por lo que se hace necesario esbozar políticas especiales para esta población.

En este sentido, la propuesta debe considerar la importancia del desarrollo social o humano, principalmente en las regiones, y para las personas que tienen carencias en sus condiciones de vida. Este desarrollo social se consolida en términos del acceso a otros bienes y servicios como educación, salud, vivienda, etc. Para esto, es necesario que se realicen inversiones en infraestructura, productividad, vías de comunicación y en sectores económicos clave como la construcción (para aumentar la demanda, la producción y el empleo) y que se desarrollen programas de alimentación y de acceso a bienes y servicios menos costosos (López, 2007; Robison y col., 2003).

Es por eso que el tratamiento académico y los programas y proyectos del estado para disminuir la pobreza y aumentar las condiciones de vida de la población, deben partir de una adecuada caracterización conceptual de la pobreza, del desarrollo social, del crecimiento y de la inequidad, como variables que pueden ser independientes en una economía (Robison y col., 2003). Creemos que esta concepción es aplicable en el contexto del presente trabajo, pues la erradicación de la obesidad en la pobreza debe entender la complejidad de esta última que, tal vez, se traduzca también en la complejidad de la relación entre la obesidad y el nivel socioeconómico.

3 Los principales cambios desde el punto de vista energético y dietético habían sido abordados con anterioridad (cambios más relevantes en los patrones alimentarios). 
Los estudios epidemiológicos más antiguos que consideraban los determinantes sociales de la salud enfocaban, principalmente, las asociaciones entre la desnutrición y las enfermedades transmisibles con la pobreza. La obesidad y las enfermedades crónicas, eran relacionadas, principalmente, con el bienestar económico. Estas concepciones no tienen más vigencia en los días actuales, manifestándose una relación bastante compleja y multifactorial, tanto en los países desarrollados como subdesarrollados y de diferentes maneras (Peña y Bacallao, 2005; Albala y Vio, 2006).

La obesidad y la desnutrición coexisten en niños de las mismas regiones y estratos sociales, en las mismas familias (madres obesas y niños desnutridos) y aun en el mismo individuo (obesidad con retraso crónico de crecimiento) (Peña y Bacallao, 2005)4. En las áreas metropolitanas periféricas urbanas de los países subdesarrollados de la región es común encontrar familias pobres con un padre de baja talla, obeso, hipertenso y con antecedentes de desnutrición en la niñez; la madre anémica, probablemente obesa y de estatura baja, y hijos parasitados que padecen procesos infecciosos frecuentes y tienen retraso del crecimiento (Peña y Bacallao, 2005; Albala y Vio, 2006). Por eso, considerar la obesidad como una condición opuesta a la desnutrición y asociada a la opulencia, no solo es equivocado, sino que puede tener consecuencias graves sobre el abordaje de los problemas nutricionales (Peña, 2001; Gotthelf y col., 20043; Amador, 1996).

En los pobres, la obesidad se asocia a episodios de desnutrición en la edad temprana, incluso durante la vida intrauterina. Estas carencias generan una respuesta de adaptación al escaso aporte de energía que, en sucesivas etapas de mayores ingestiones, pueden producir como resultado un aumento de peso. En estos casos, la obesidad se relaciona con ingesta energética superior a las necesidades y deficiente en nutrientes esenciales, como aminoácidos y micronutrientes. Los pobres seleccionan alimentos pobres en fibras y ricos en carbohidratos complejos, azúcares y grasas (especialmente ácidos grasos trans, ácidos grasos saturados y colesterol) que, aunque les impiden gozar de una nutrición adecuada, satisfacen su apetito, se integran bien a su patrón de consumo tradicional y a sus pautas de comensalismo. El consumo de comidas rápidas, caracterizadas por una elevada densidad energética y baja calidad nutricional, presenta gran influencia en estos contextos. La salud de este grupo de obesos pobres se deteriora todavía más, dado su limitado acceso a los servicios adecuados de asistencia médica (Peña, 2001) ${ }^{1}$.

Los estudios entre las condiciones sociales y la obesidad indican una relación compleja y diferente en contextos socioeconómicos distintos. Las evidencias señalan tanto relaciones directas como inversas, indicando interacciones complicadas y múltiples entre el conjunto de factores biológicos, sociales, culturales, comerciales, etc. Peña y Bacallao (2005) resumen esta diversidad, manifestando que establecer conclusiones definitivas sobre la asociación entre la obesidad y la condición socioeconómica resulta muy difícil por dos razones: i) la asociación puede variar de un país a otro o de una región a otra en el mismo país, ii) la asociación puede estar confundida por factores culturales, ecológicos o sociales. Los autores indican que en algunos países de la región la asociación entre obesidad y condición socioeconómica se asemeja a la que exhiben los países desarrollados y que en otros la relación puede ser inversa. Adicionalmente, refieren que, probablemente, ese patrón variable de comportamiento se esté encaminando hacia un patrón de asociación inversa siguiendo el ritmo y modelo de transición epidemiológica y nutricional característico de cada país.

\section{Causas de la Obesidad en la Pobreza}

La etiología multifactorial de la obesidad comprende el análisis de un conjunto de factores genéticos y metabólicos, socioculturales y simbólicos, psíquicos, ambientales y relacionados a los estilos de vida. La causa básica de la obesidad refiere el factor alimentario, caracterizado por una ingestión de energía mayor que el gasto energético, asociado a los nuevos estilos de vida (hábitos alimentarios y sedentarismo) (Ferreira y Wanderley, 2007; Batista Filho, 2003). Las características de la obesidad pueden ser diferentes entre individuos de distintos niveles socioeconómicos de un mismo país, así como también entre los individuos

4 GOTTHELF, S.; ARAMAYO, M. E.; ALDERETE, S. Coexistencia de obesidad y desnutrición en familias pobres. 2004. Monografía (Postgrado a distancia en obesidad) - Universidad de Favaloro, Buenos Aires, 2004. 
más pobres y más ricos de diversos países, sean éstos desarrollados o en desarrollo'.

Para poder analizar, de forma crítica, las diferencias anteriores, es importante que se tenga presente la acción de factores de diferente naturaleza:

\section{Factores Alimentarios}

Las poblaciones urbanas de diversos países han modificado su régimen alimenticio a expensas del aumento en el consumo de grasas, azucares y de la disminución en el consumo de fibra. Los precios elevados de las frutas, vegetales frescos y de otros alimentos de alta calidad nutricional, los hacen inaccesibles para los grupos de ingresos más bajos. Por su parte, la industria alimenticia favorece ese comportamiento al segmentar la oferta y comercializar productos masivos de mayor contenido en grasas, azucares y de baja calidad nutricional (deficientes en nutrientes esenciales). Además, estos alimentos se caracterizan por su alto poder de saciedad, sabor agradable y bajo costo. Todo esto hace con que estos alimentos sean socialmente aceptables y preferidos por los grupos más pobres (Batista Filho, 2003; Gutiérrez, 2004).

\section{Factores Genéticos de Adaptación}

Según la hipótesis del "genotipo de ahorro", los obesos pobres podrían desarrollar un mecanismo de adaptación metabólica, en el cual las poblaciones expuestas a un consumo inadecuado o fluctuante de alimentos generan formas adaptativas para lograr un nivel alto de eficiencia en el uso de la energía y el depósito de grasa. Cuando esos grupos logran disponer de alimentos en forma regular y mantienen esas formas de adaptación, puede presentarse un aumento en los depósitos de grasa y, por consiguiente, exceso de peso. Esta teoría revierte especial importancia desde el punto de vista generacional, pues expresa los plausibles cambios que pueden darse en poblaciones de diferentes generaciones, manifestándose de forma más clara en algunos individuos cuando comparados con otros y pudiendo, aunque no necesariamente, estar asociado al nivel socioeconómicoํ.

\section{Desnutrición Intrauterina}

La teoría de Barker hace referencia al impacto de las condiciones de vida intrauterina sobre la aparición de enfermedades en la vida adulta. Distingue que la nutrición que antecede y procede al nacimiento, así como experiencias adversas en el medio intrauterino, ejercen una influencia importante en el desarrollo futuro de enfermedades degenerativas, pues condicionan una mayor susceptibilidad a los factores ambientales dependientes de la alimentación y del estilo de vida. El autor enfatiza que un mal progreso fetal, evaluado por el peso al nacer, sería un preeditor del futuro desarrollo de obesidad, hipertensión, hiperlipidemias, alteraciones en la coagulación y mortalidad cardiovascular (Barker y col., 2002, p. 1235; Barker, 2004).

\section{Factores Socioculturales}

El ambiente violento, agresivo y inseguro que se vive principalmente en las zonas periféricas urbanas, impide que la población pobre tenga estilos de vida saludables. Esto dificulta la práctica de ejercicios físicos en forma sistemática y el derecho de recibir informaciones sobre nutrición, actividad física y salud. Las inequidades en el acceso a los mensajes de promoción de la salud, a la educación sanitaria y a los servicios adecuados de atención de la salud, impiden conocer la importancia de los cambios de comportamiento necesarios para lograr un modo de vida más sano. Por otra parte, en ciertas culturas primitivas, o poco desarrolladas, el sobrepeso es visto como un signo de prestigio social y de riqueza. Además, la acumulación excesiva de grasa, sobre todo en las mujeres, puede ser percibida como un signo de belleza y de atractivo sexual (Peña y Bacallao, 2005; Aguirre, 2006; Sobal y Stunkard, 1989, p. 260).

\section{Aculturación a Distancia}

La aculturación a distancia es un término que refiere el resultado del contacto de los países de América Latina y el Caribe con los patrones culturales de los países desarrollados, principalmente la influencia ejercida por la rapidez en los avances de la ciencia y de la industria de comunicación. Es un proceso en el cual la industria del consumo despierta en los individuos la necesidad de incorporar algunos elementos de la imagen proyectada y los impulsa a adoptar hábitos y modos de vida inadecuados. Los grupos más prósperos consiguen adaptarse más fácilmente a esos cambios. Por el contrario, los más pobres padecen el conflicto entre sus capacidades y la imagen ideal con la que se identifican (Peña y Bacallao, 2005; Popkin, 200o; Aguirre, 2006). 


\section{Género}

El género es otro aspecto importante en la prevalencia de obesidad entre mujeres de poca condición socioeconómica. Las mujeres tienen oportunidades más limitadas, cargas sociales más pesadas, una imagen subvalorada de su cuerpo y, tradicionalmente, se encuentran subordinadas socialmente a los hombres. Todo esto representa un aumento de la susceptibilidad femenina al conjunto de influencias desfavorables relacionadas con la obesidad en la pobreza (Popkin, 2000; Aguirre, 2006) ${ }^{1}$.

\section{Migración}

Las migraciones de los pobladores rurales hacia las periferias urbanas y de países más pobres hacia otros en mejor situación, han producido cambios importantes en las condiciones y el modo de vida de dichos grupos. Este hecho relaciona la migración con la obesidad si consideramos que, en general, la tendencia de aumento de la obesidad en adultos a nivel mundial puede explicarse por el proceso de urbanización y aumento en la expectativa de vida, disminución del gasto energético relacionado con el avance tecnológico, la urbanización y el aumento progresivo en el consumo de grasa y de la densidad energética provenientes de alimentos industrializados más accesibles en el mercado. Por otro lado, cabe señalar que también muchos inmigrantes, huyendo de la pobreza o en la búsqueda de trabajo, pueden tener como destino lugares que no poseen la infraestructura urbana (agua, alcantarillado, habitación, etc.), social (educación y salud) y económica (oportunidades de empleo, abastecimiento alimentario, etc.) para absorberlos y proporcionarles condiciones dignas de vida (Popkin, 200o; Figueroa Pedraza, 2004a).

\section{Factores Socioeconómicos}

La asociación entre obesidad y condición socioeconómica ha sido confirmada a través de varios estudios en diferentes países. La relación se ha manifestado de forma variable y compleja, presentándose gradientes socioeconómicos heterogéneos y de acuerdo al ritmo y al modelo de transición epidemiológica y nutricional característicos de cada país. Así, la literatura refleja resultados en los cuales la condición socioeconómica y obesidad pueden estar relacionadas tanto de forma directa como de forma inversa. Por otra parte, se manifiesta, también, la importancia que el sexo ejerce en este contexto (Gutiérrez Fisac, 1998, p. 347; Rosengren y Lissner, 2008, p. 260).

\section{Relación entre Situación Socioeconómica y Obesidad en Diferentes Contextos}

La relación entre la condición socioeconómica y la obesidad puede estar influenciada por el estadio de desarrollo social y económico de los diferentes países. En los países desarrollados de Europa y de América se ha encontrado, en casi todos los estudios, asociación inversa entre el nivel socioeconómico y la obesidad, independiente de la forma adoptada para evaluar la condición socioeconómica (nivel educacional, ingresos, ocupación laboral) (Song, 2006, p. 112). En los países en desarrollo los estudios han resultado tanto en asociaciones positivas como negativas. Los resultados cambian como consecuencia del nivel de desarrollo y del indicador de condición socioeconómica utilizado. En general, un menor nivel socioeconómico se ha asociado con mayor sobrepeso/obesidad en mujeres de países más desarrollados cuando se utilizan los indicadores educación y ocupación, mientras que la asociación positiva se ha establecido en mujeres de países con bajo y mediano desarrollo cuando se utilizan los indicadores de renta y bienes materiales (McLaren, 2007, p. 29).

A pesar de que muchos estudios muestren en sus resultados una relación directa entre la situación socioeconómica y la obesidad, varios autores indican la importancia de no considerar este hecho de forma aislada ni como una relación causal, pues la complejidad del proceso se asocia con su evolución temporal, implicando en cambios que pueden resultar en un mayor predominio de la obesidad en las clases más pobres, principalmente entre las mujeres. Así, por el contrario, la asociación directa entre el nivel socioeconómico y la obesidad, parece apoyar la hipótesis de que la transición en el desarrollo económico está acompañada de cambios en el estilo de vida (patrones alimentario y de actividad física) que pueden explicar esa asociación. Con el desarrollo socioeconómico desaparecen los factores que protegen a los pobres contra la obesidad (desaparece la escasez de alimentos y el gran gasto energético de los más pobres) mientras que 
se mantienen y fortalecen los que protegen a las clases de mayor poder adquisitivo (las personas con mejores condiciones económicas adquieren alimentos más apropiados y conservan valores culturales relacionados al cuidado del cuerpo como símbolo de status social) (Rosengren y Lissner, 2008, p. 260; Pinheiro y col., 2004, p. 523; Song, 2006, p. 112).

Por otro lado, Song (2006) reflexiona sobre la forma de interpretar esos resultados, indicando que un aspecto metodológico fundamental en cualquier estudio sobre obesidad y condición socioeconómica es la conveniencia de ajustar algunos factores (el consumo y el gasto energético funcionan como mediadores, por lo que no se deben ajustar en estos tipos de estudios) para lograr resultados no sesgados. Como ejemplo, cita la actividad física (tiempo libre y tiempo dedicado a alguna actividad física), estrechamente relacionada con el nivel de gasto energético.

De lo anteriormente expresado podemos concluir que encontramos dos perfiles diferentes al estudiar las relaciones entre obesidad y situación socioeconómica: I - Asociación directa en la cual los individuos de mayor nivel socioeconómico tienen, en general, una mayor frecuencia de obesidad.

II - Asociación inversa en la cual los individuos de menor nivel socioeconómico tienen, en general, una mayor frecuencia de obesidad.

\section{Explicaciones de la Asociación Directa entre Obe- sidad y Condición Socioeconómica}

La mayor frecuencia de obesidad en las clases sociales altas, principalmente en los países en desarrollo, puede ser explicada por dos factores (Gutiérrez Fisac, 1998, p. 347; Song, 2006, p. 112):

1. Económico: La menor disponibilidad de alimentos en los países en desarrollo, que afecta los niveles socioeconómicos más bajos, produce una eficiencia metabólica que puede resultar en un cúmulo excesivo de grasa en relación al ingreso energético por exposición a condiciones favorecidas con el desarrollo socioeconómico (cantidad suficiente de alimentos y trabajos menos intensos).

2. Cultural: Se relaciona con la valoración social de la obesidad cuando el sobrepeso es visto como un signo de prestigio social, riqueza, belleza y atractivo sexual, estos dos últimos sobre todo en las mujeres.

\section{Explicaciones de la Asociación Inversa entre Obe- sidad y Condición Socioeconómica}

Muchos son los factores que influyen en la asociación inversa nivel socioeconómico - obesidad, presente tanto en los países desarrollados como en aquellos en desarrollo. Estos podrían ser agrupados de la siguiente manera (Gutiérrez Fisac, 1998, p. 347; Pinheiro y col., 2004, p. 523; McLaren, 2007, p. 29):

1. Principales factores implicados en el metabolismo graso (principales componentes del balance energético): Potenciales moderadores de la relación nivel socioeconómico - obesidad

\subsection{Ingreso: Ingreso energético \\ 1.2. Gasto: Ejercicio físico}

2. Sexo y edad: Refiere las variaciones (resultados (in)consistentes) sobre la magnitud del problema cuando abordadas estas variables

3. Movilidad Social (cambio del nivel socioeconómico de los individuos a lo largo de su ciclo vital): Único factor en el que la obesidad modifica (reduce) el nivel socioeconómico

4. Otros factores

4.1. Culturales

4.2. Psicosociales

4.3. Herencia

4.4. Efectos de la urbanización y de la industrialización en los estilos de vida de las poblaciones rurales

\section{Ingreso Energético}

La importancia del ingreso energético en el peso corporal es innegable. La acumulación de grasa en el organismo está mediada por el ingreso y el gasto energético. Un aumento del ingreso y/o un descenso del gasto energético producirá un aumento de la grasa en el organismo (Batista Filho, 2003).

Sin embargo, estudios en humanos, basados en datos poblacionales, muestran resultados inconsistentes relacionados al hecho anterior. Estudios de base individual no han conseguido establecer una relación entre el ingreso energético auto-referido y el peso corporal. En relación al contenido de grasa en la dieta grasa y el peso corporal tampoco se ha establecido una asociación clara a través de estudios epidemiológicos. Por su parte, estudios ecológicos y ensayos clínicos randomizados han mostrado la reducción del peso como consecuencia de restricción energética y de disminución del conteni- 
do de grasa en la dieta (Gutiérrez Fisac, 1998, p. 347; Lissner y Heitmann, 1995, p. 79).

Estas inconsistencias en la asociación entre el ingreso energético y la obesidad han sido atribuidas a varias causas, entre las que se destacan: i) la posibilidad de que los instrumentos disponibles para evaluar el consumo de alimentos en estudios epidemiológicos no sean los más adecuados, la disponibilidad de datos adecuados continúa siendo la principal restricción en los métodos de evaluación del consumo de alimentos; ii) la dieta medida en estudios epidemiológicos puede no representar el consumo a largo plazo, ya que, aun tratándose de estudios longitudinales, el consumo de alimentos se mide en determinados momentos, olvidando ciertos cambios que pueden influir en el peso; iii) la importancia de cierta predisposición individual en la asociación ingreso energético-obesidad (Gutiérrez Fisac, 1998, p. 347; Pérez Rodrigo, 1994; Figueroa Pedraza, 2004b, p. 69).

Por lo tanto, puede afirmarse la necesidad de más estudios epidemiológicos con diseños adecuados que posibiliten verificar la influencia del contenido energético de la alimentación en la relación nivel socioeconómico - obesidad.

\section{Ejercicio Físico}

El balance energético es una función del ingreso (componente anterior) y el gasto energético. Varios factores como la tasa metabólica basal, la termogénesis y la actividad física determinan el gasto energético y, por lo tanto, influyen en la cantidad de grasa acumulada por el organismo. Dos razones hacen centrar la atención en el ejercicio físico cuando de gasto energético se trata. En primer lugar, la tasa metabólica basal y la termogénesis son factores de difícil medición en estudios poblacionales; en segundo lugar, la actividad física es el componente más variable del gasto energético por lo que su reducción produce un aumento de la grasa acumulada en el organismo (Batista Filho, 2003; Lissner y Heitmann, 1995, p. 79; Sichieri y col., 1994, p. 793).

Los individuos que realizan más ejercicio físico tienen un peso corporal menor, incluso controlando el efecto de otros factores con influencia sobre la obesidad. Cuando colocamos el nivel socioeconómico, también como variable de análisis, se observan claras diferencias en la actividad física; los individuos de más alto nivel socioeconómico son los que realizan más ejercicio físico (Gutiérrez Fisac, 1998, p. 347).

\section{Sexo y Edad}

A pesar de que son necesarios más estudios para clarear la naturaleza exacta de la relación entre la situación socioeconómica y la obesidad, considerando las variaciones por sexo y edad, la asociación inversa ha sido conclusiva en revisiones sistemáticas sobre el tema (Shrewbury y Wardle, 2008, p. 275; Ball y Crawford, 2005, p. 1987; Monteiro y col., 2004, p. 940) para las siguientes condiciones:

- En los países desarrollados, la obesidad está inversamente relacionada con el nivel socioeconómico en mujeres, y de forma menos consistente en hombres;

- En los países subdesarrollados, los estudios han reportado conclusiones más firmes, apuntando la asociación inversa, para el caso de las mujeres, en el caso particular de los hombres emergen resultados más inconsistentes;

- El estadio de desarrollo (Producto Interno Bruto) de cada país influencia la mayor o menor probabilidad de encontrar resultados indicando asociación inversa, siendo la probabilidad mayor en los países más desarrollados (mayor Producto Interno Bruto);

- El cambio de asociación directa para asociación inversa, ocurre en una fase anterior del desarrollo económico (menor Producto Interno Bruto) para el caso de las mujeres con bajo nivel socioeconómico cuando comparado con los hombres;

- A pesar de estudios que indican relación inconsistente entre el nivel socioeconómico y la adiposidad infantil, los resultados constatan una relación predominantemente inversa.

\section{Movilidad Social}

El concepto de movilidad social surge a la luz de estudios que indican que la obesidad es un estigma que produce discriminación social, haciendo que los individuos más obesos desciendan en la escala social, o sea, existencia de un mecanismo en el cual la obesidad ejerce un efecto negativo sobre la clase social. De esta manera, la relación de la obesidad con la condición socioeconómica, además de compleja y multifactorial, también se muestra bi-direccional: el bajo nivel socioeconómico determinaría el aumento de la prevalencia 
de obesidad, en cuanto la alta prevalencia de obesidad contribuiría para una disminución del nivel socioeconómico, por limitaciones funcionales, estéticas y culturales. Como resultado de la movilidad social, las mujeres que cambian su clase social muestran prevalencias de obesidad del grupo que adhieren, asociación que no se encuentra en los hombres. Algunos estudios también muestran que la presencia de obesidad en la adolescencia puede producir consecuencias sociales en la edad adulta, como una menor frecuencia de casamientos, menores ingresos y menor escolaridad, particularmente en el sexo femenino (Gutiérrez Fisac, 1998, p. 347; Parsons y col., 1999, p. S1).

\section{Factores Culturales}

Algunos trabajos han intentado penetrar en las representaciones sociales, dimensiones simbólicas y culturales que el cuerpo - obesidad y desnutrición - tiene como expresión física del estado nutricional. El cuerpo representa una categoría responsable por el acceso al mundo, donde está presente el aspecto existencial, el modo de ser y de manifestarse en el mundo (Tonial, 2001).

Existen dos visiones bastante diferentes relacionadas a la obesidad. En las sociedades más atrasadas el sobrepeso es visto como un signo de prestigio social y de riqueza. En las sociedades desarrolladas la obesidad es un estigma asumido que coloca una correlación negativa entre peso y satisfacción (a mayor peso, mayor insatisfacción personal con el cuerpo). De esta forma, la opinión acerca del sobrepeso varía con el nivel socioeconómico y tiene, por lo tanto, una gran influencia en la relación nivel socioeconómico-obesidad. Los individuos de mayor nivel socioeconómico son los que con más frecuencia creen que su peso está por encima del ideal, aun teniendo un peso dentro del rango de lo normal (Aguirre, 2006; Tonial, 2001).

Toda esta evidencia ha llevado a sugerir la hipótesis de que la existencia de presiones familiares y sociales para mantener una imagen corporal aceptable y acorde con los valores sociales dominantes es responsable, en parte, de la distribución social de la obesidad (Gutiérrez Fisac, 1998, p. 347). Este hecho se refuerza si se realizan consideraciones de género. Las normas culturales hacen a las mujeres victimas de un proceso de segregación urbana, que limita sus oportunidades de actividad y fuentes de estimulación cultural, y a su vez, disminuye el valor social de sus cuerpos, limitan- do su papel social únicamente a la procreación (Peña y Bacallao, 2006). Así, este fenómeno podría explicar también la desventaja del género femenino en la asociación nivel socioeconómico-obesidad.

\section{Factores Psicosociales}

La importancia de los cuidados en el estado nutricional es tan crucial que constituye una de las tres causas básicas de los problemas nutricionales. El término cuidados se refiere a la capacidad de la familia y de la comunidad para dedicar tiempo, atención, ayuda y conocimientos prácticos para cubrir las necesidades físicas, mentales y sociales de las personas nutricionalmente vulnerables, en particular los niños en crecimiento. Estos cuidados redundan en el uso óptimo de los recursos humanos, económicos y sociales. A pesar de que tanto las madres como su educación son el factor principal de los cuidados, es necesario el apoyo de toda la familia al ser ésta la unidad social económica de mayor responsabilidad para el bienestar nutricional; esto hace imprescindible considerar las funciones, conocimientos, limitaciones de tiempo, recursos y motivaciones de los miembros de la familia (Conferência Internacional sobre Nutrición, 1992).

Algunos estudios han mostrado que la existencia de un buen soporte social (indicado por el estado civil, por ejemplo) de la madre, o cuidador principal, tiene un efecto protector en relación a la obesidad en la infancia. Se piensa que el soporte social esté relacionado con el nivel socioeconómico de forma positiva. Así, los cuidados podrían estar mediando en la asociación nivel socioeconómico-obesidad. En general, han sido pocos los estudios desarrollados para evaluar la influencia de los cuidados infantiles en la obesidad adulta considerando también la importancia de factores sociales como el tamaño de la familia, la estructura familiar, el número de personas que viven en la misma casa, la higiene y la negligencia paterna (Parsons y col., 1999, p. S1).

\section{Herencia}

Cuando hablamos de herencia casi siempre nos concentramos en aspectos puramente genéticos y dejamos de un lado su aspecto social, sumamente importante en los análisis de desigualdad social en salud. La contribución relativa de los factores genéticos y de los estilos de vida heredados en la obesidad permanecen largamente desconocidos (Parsons y col., 1999, p. S1). 
Es bien argumentado en la literatura el importante componente genético de la obesidad. Existe una asociación entre la obesidad de los padres y la obesidad de los hijos. Así mismo, estudios han mostrado que el nivel socioeconómico de los padres está relacionado con el de los hijos, indicando que hay cierta transmisión hereditaria de algunos factores (educacionales, actitudes y conocimientos sobre alimentación, imagen corporal, etc.) que pueden condicionar el nivel socioeconómico alcanzado por el individuo o ciertos atributos relacionados al mismo como el bienestar psicológico, social y material (Barker, 2004; Sobal y Stunkard, 1989, p. 260).

Parsons y colaboradores (1999), en revisión sistemática sobre los preeditores infantiles de la obesidad adulta colocan otros aspectos que también merecen ser destacados:

- Existe una fuerte y consistente relación entre el nivel socioeconómico en la infancia y el aumento de la adiposidad en la vida adulta;

- Existen evidencias sugestivas del efecto protector de la actividad física infantil y el riesgo de ser obeso cuando adulto;

- Existen evidencias claras sobre la influencia del sobrepeso al nacer en la adiposidad, siendo la consistencia menor cuando se trata la confusión por edad gestacional, adiposidad parental y grupo social.

\section{Efectos de la Urbanización y de la Industrialización en los Estilos de Vida de las Poblaciones Rurales}

Las presiones económicas y los incentivos que promueven la migración rural para áreas periféricas urbanas (más centros de salud, escuelas, canales de comunicación y más tiempo libre para actividades recreativas) determinan un mayor riesgo de obesidad. Por lo general, poblaciones que antes de migrar tenían actividad física con alto desgaste, asociada con la producción agrícola, se tornan poblaciones urbanas marginales con menos trabajo físico; dieta desequilibrada, rica en grasa, energía y bebidas adulzadas; mayor consumo de alcohol y estilo de vida sedentario. Con base en la hipótesis de que los seres humanos poseen tendencia natural a mantener el equilibrio energético, las alteraciones que promueven hábitos dietéticos inadecuados y que no estimulan la realización de actividad física aumentan el riesgo de obesidad y de otras enfermedades asociadas (Peña y Bacallao, 2006).

\section{Conclusiones}

- La obesidad es el resultado de la conjunción de factores biológicos, genéticos y ambientales, así como de los estilos de vida, sobre todo la inactividad física, el sedentarismo creciente y el desequilibrio entre las necesidades energéticas y la dieta.

- El estudio de tendencias de la prevalencia y diferencias sociales actuantes en la obesidad, muestran un progresivo y acelerado aumento.

- La tendencia de aumento de la obesidad puede ser justificada por toda una serie de cambios en el régimen alimentario (preferencias por alimentos procesados, pobres en fibras y con altos contenidos de carbohidratos simples y grasas saturadas), el patrón de trabajo, la actividad física y tipo de recreación.

- Muchas de las personas que experimentan estos cambios son emigrantes en busca de mejores condiciones de vida. La mayoría de ellos seguirán en ciertos grados de pobreza, dentro de la estructura social, en una situación de incapacidad económica para acceder a alimentos saludables.

- Existen evidencias científicas suficientes sobre la relación entre la obesidad y la situación socioeconómica. No obstante, es difícil establecer conclusiones definitivas, pues la asociación se muestra inconsistente en diferentes contextos, siendo necesario estudios para una mejor caracterización, si bien está claro que tanto factores biológicos como sociales y culturales influyen en la obesidad y en el nivel socioeconómico.

- El nivel socioeconómico condiciona la presencia de obesidad de forma independiente, tanto de factores genéticos como de factores ambientales, diferentes del nivel socioeconómico.

- Son varios los factores que ejercen cierta influencia en la relación nivel socioeconómico- obesidad. La mayoría de ellos son mediadores en dicha relación.

- Tanto la obesidad podría tener consecuencias sociales en el individuo, como el nivel socioeconómico podría condicionar la presencia de obesidad. Diversos factores, podrían mediar y complicar el establecimiento de una relación causal; el ejercicio físico, la dieta, los factores psicológicos, la herencia y los factores socioculturales podrían influir, de forma no bien conocida, en la asociación nivel socioeconómico-obesidad. Si la movilidad social es el factor más relevante, es la 
obesidad la que condicionaría la posición social del individuo.

- Los argumentos tratados en el artículo son suficientes para justificar la importancia del problema en Latinoamérica. De esta manera, debe representar un desafío, tanto para los investigadores como para aquellos que toman decisiones, entre otros actores, colocando la obesidad en condiciones de pobreza como una prioridad en sus agendas de trabajo.

- Los resultados sugieren la necesidad de estudios que posibiliten el conocimiento de las particularidades en el establecimiento de la situación socioeconómica como factor de exposición importante en el desarrollo de adiposidad.

- La asociación inversa entre el nivel socioeconómico y la obesidad se manifiesta de forma diferente dependiendo del desarrollo alcanzado. Es inadmisible continuar considerando que la obesidad en los países en desarrollo sea considerada una enfermedad apenas de los grupos de mayor nivel socioeconómico. Las minorías y aquellos estratos de la población con menor nivel educativo y socioeconómico pueden ser los más afectados por la obesidad en los países en desarrollo.

- Los resultados refuerzan la necesidad de prevenir la obesidad en los países subdesarrollados; aumentar el acceso a la información sobre alimentación y nutrición sobre los determinantes y consecuencias de la obesidad; implementar estrategias públicas que aborden el ambiente físico, económico y sociocultural, posibilitando una mejor alimentación y incorporación de la actividad física. El enfoque debe estar dirigido a todas las clases sociales, considerando de forma particular los grupos socioeconómicos más desfavorecidos.

\section{Referencias}

AGUIRRE, P. Aspectos socioantropológicos da obesidade na pobreza. In: PEÑA, M.; BACALLAO, J. (Ed.). Obesidade e pobreza: um novo desafio à saúde pública. São Paulo: Roca, 2006. p. 12-26.

ALBALA, C.; VIO, F. Obesidade e pobreza: um desafio pendente no Chile. In: PEÑA, M.; BACALLAO, J. (Ed.). Obesidade e pobreza: um novo desafio à saúde pública. São Paulo: Roca, 2006. p. 48-59.
AMADOR, M. Seminario-taller sobre obesidad y pobreza en América Latina. Revista Cubana Alimentación y Nutrición, La Habana, v. 10, n. 1, 1996. Disponível em: <http://bvs.sld.cu/revistas/ali/volio_ 1_96/ali13196.htm>. Acesso em: 6 fev. 2007.

BALL, K.; CRAWFORD, D. Socioeconomic status and weight change in adults: a review. Social Science Medicine, London, v. 6o, n. 9, p. 1987-2010, 2005.

BARKER, D. J. P. The fetal origins of obesity. In: BRAY, G. A.; BOUCHARD, C. (Ed.). Handbook of obesity. 2. ed. New York: Marcel Dekker, 2004. p. 109-116.

BARKER, D. J. P. et al. Fetal origins of adult disease: strength of effects and biological basis. International Journal of Epidemiology, Oxford, v. 31, p. 1235-1239, 2002.

BATISTA FILHO, M. Alimentação, nutrição e saúde. In: ROUQUAYROL, M. Z.; ALMEIDA FILHO, N. Epidemiologia e saúde. 6. ed. Rio de Janeiro: MEDSI, 2003. p. 309-410.

\section{CONFERENCIA INTERNACIONAL SOBRE}

NUTRICIÓN. Nutrición y desarrollo: una evaluación mundial. Roma: FAO: WHO, 1992.

DE ONIS, M.; BLÖSSNER, M. Prevalence and trends of overweight among preschool children in developing countries. American Journal of Clinical Nutrition, Houston, v. 72, p. 1032-1039, 2000.

EBERWINE, D. Globesidad: una epidemia en apogeo. Perspectivas de Salud, Washington, DC, v. 7, n. 3, p. 1-7, 2002.

FERREIRA, V. A.; MAGALHÃES, R. Obesidade no Brasil: tendências atuais. Revista Portuguesa de Saúde Pública, Lisboa, v. 24, n. 2, p. 71-81, 2006.

FERREIRA, V. A.; WANDERLEY, E. N. Obesidade: uma perspectiva plural. Ciência e Saúde Coletiva, Rio de Janeiro, [2009?]. Disponível em: <http://www.abrasco. org.br/cienciaesaudecoletiva/artigos/artigo_int. php?id_artigo=1200>. Acesso em: 11 jan. 2007.

FIGUEROA PEDRAZA, D. Padrões alimentares: da teoria à prática: o caso do Brasil. Mneme Revista de Humanidades, Caicó, v. 4, n. 8 abr./set. 2003. Disponível em: <http://www.cerescaico.ufrn.br/ mneme/sobre/contato.html>. Acesso em: 11 jan. 2007. 
FIGUEROA PEDRAZA, D. Consumo de alimentos como resultante de la seguridad alimentaria y nutricional en Brasil. Perspectivas en Nutrición Humana, Medellín, v. 11, p. 69-86, 2004a.

FIGUEROA PEDRAZA, D. Seguridad alimentaria y nutricional en Brasil. Revista Salud Pública y Nutrición, Monterrey, v. 5, n. 3, 2004b. Disponível em: <http://www.respyn.uanl.mx/v/3/ensayos/sa_en brasil.htm>. Acesso em: 6 fev. 2007.

GUTIÉRREZ, D. La obesidad en la pobreza: una perspectiva de salud internacional en prevención. Monterrey, 2004. Disponível em: <http://www.respyn. uanl.mx/especiales/ee-9-2004/index.html>. Acesso em: 6 set. 2006.

GUTIÉRREZ FISAC, J. L. Obesidad y nivel socioeconómico. Medicina Clínica, Barcelona, v. 110, n. 9, p. 347-355, 1998.

GUTIÉRREZ FISAC, J. L.; REGIDOR, E.; RODRÍGUEZ, C. Trends in obesity differences by educational level in Spain. Journal of Clinical Epidemiology, New York, v. 49, p. 351-354, 1996.

HOLDAGO, B.; MARTÍNEZ, M.; MARTÍNEZ, A. Obesidad. In: MARTÍNEZ, A.; ASTIASARÁN, I.; MADRIGAL, H. Alimentación y salud pública. 2. ed. Madrid: McGraw-Hill, 2002. p. 189-197.

LÓPEZ, H. La pobreza y la desigualdad en Colombia. Bogotá: Conciudadana, 2007.

LISSNER, L.; HEITMANN, B. L. Dietary fat and obesity: evidence from epidemiology. European Journal of Clinical Nutrition, London, v. 49, p. 79-9o, 1995.

MCLAREN, L. Socioeconomic status and obesity. Epidemiology Review, Baltimore, v. 29, p. 29-48, 2007.

MONTEIRO, C. A. et al. Socioeconomic status and obesity in adult populations of developing countries: a review. Bulletin of the World Health Organization, Geneva, v. 82, n. 12, p. 940-946, 2004.

PARSONS, T. J. et al. Childhood predictors of adult obesity: a systematic review. International Journal of Obesity and Related Metabolic Disorders, England, v. 23, p. S1-S107, 1999. Supplement 8.
PÉREZ RODRIGO, C. Fuentes de error en la evaluación del consumo de alimentos. In: SERRA MAJEM, L. L.; ARANCETA BARTRINA, J.; MATAIX VERDÚ, J. Nutrición y salud pública: métodos, bases científicas y aplicaciones. Barcelona: Masson, 1994. p. 168-172.

PEÑA, M. Obesidad y pobreza en América Latina. Disponível em: <http://www.saota.org.ar/RevistaObesidad-Abril-2001/sumario.htm>. Acesso em: 25 set. 2006.

PEÑA, M.; BACALLAO, J. (Ed.). Obesidade e pobreza: um novo desafio à saúde pública. São Paulo: Roca, 2006.

PEÑA, M.; BACALLAO, J. La obesidad en la pobreza: un problema emergente en las Américas. Revista Futuros, México, DF, v. 3, n. 10, 2005. Disponível em: <http://www.revistafuturos.info/futuros_10/ obesidadı.htm>. Acesso em: 14 nov. 2006.

PEÑA, M.; BACALLAO, J. La obesidad y sus tendencias en la región. Revista Panamericana Salud Pública, Washington, DC, v. 10, n. 2, p. 75-78, 2001.

PINHEIRO, A. R. O.; FREITAS, S. F. T.; CORSO, A. C. T. Uma abordagem epidemiológica da obesidade. Revista de Nutrição, Campinas, v. 17, n. 4, p. 523-533, 2004 .

POPKIN, B. M. The nutrition transition and its implications in lower-income countries. Public Health Nutrition, Cambridge, v. 1, p. 5-21, 1998.

POPKIN, B. M. Lograr la seguridad alimentaria y nutricional urbana en el mundo en desarrollo: la urbanización y la transición nutricional. Washington, DC: IFPRI, 2000.

POPKIN, B. M. The nutrition transition and obesity in the developing world. Journal of Nutrition, Oxford, v. 131, p. 871S-873S. 2001. Supplement.

RIVERA, J. et al. Epidemiological and nutritional transition in Mexico: rapid increase of noncommunicable chronic diseases and obesity. Public Health Nutrition, Cambridge, v. 5, n. 1A, p. 123-128, 2002. 
ROBISON, L.; SILES, M.; SCHMID, A. El capital social y la reducción de la pobreza: hacia un paradigma maduro. In: ATRIA, R. et al. (Comp.). Capital social y reducción de la pobreza en América Latina y el Caribe: en busca de un nuevo paradigma. Santiago de Chile: CEPAL, 2003. p. 51-114.

ROSENGREN, A.; LISSNER, L. The sociology of obesity. Obesity and Metabolism, Basel, v. 36, p. 260-270, 2008.

SHREWBURY, V.; WARDLE, J. Socioeconomic status and adiposity in childhood: a systematic review of cross-sectional studies 1990-2005. Obesity, Silver Spring, v. 16, n. 2, p. 275-284, 2008.

SICHIERI, R. et al. High temporal, geographic and income variation in body mass index among adults in Brazil. American Journal of Public Health, Stanford, v. 84, p. 793-798, 1994.
SOBAL, J.; STUNKARD, A. J. Socioeconomic status and obesity: a review of the literature. Psychological Bulletin, Washington, DC, v. 105, n. 2, p. 260-275, 1989.

SONG, Y. M. Commentary: varying relation of socioeconomic status with obesity between countries at different stages of development. International Journal of Epidemiology, v. 35, n. 1, p. 112-113, 2006.

TONIAL, S. Desnutrição e obesidade: faces contraditórias na miséria e na abundância. Recife: IMIP, 2001.

UAUY, R.; ALBALA, C.; KAIN, J. Obesity trends in Latin America: transiting from under to overweight. Journal of Nutrition, Oxford, v. 131, p. 893S-899S, 2001. Supplement. 